\title{
A Hybrid 3D Quantum Mechanical Simulation of FinFETs and Nanowire Devices
}

\author{
Xue Shao and Zhiping Yu \\ Institute of Microelectronics, Tsinghua University, Beijing 100084, China \\ shaox97@mails.tsinghua.edu.cn
}

\begin{abstract}
In this paper, we have carried out a numerical simulation of FinFETs. The model is based on 1D non-equilibrium Green's function (NEGF) along the channel and 2-D Schrödinger equation in the confined cross section and provides insights into the performance of FinFETs with ultra small channel cross section. The simulation results of FinFETs show normal $I$ - $V$ characteristics with great potential in scalability even when the gate length is below $5 \mathrm{~nm}$ with 2-by- $2 \mathrm{~nm}$ channel cross section.
\end{abstract}

\section{Introduction}

Double gate (DG) or triple gate (commonly known as FinFETs) MOSFETs possess superior gate controllability in both off and on states. Their (for DG, it is restricted to vertical type only) compatibility with the planar process makes them the strong contender in sub-65nm CMOS technology nodes. There has been extensive research work performed regarding DG-FETs, e.g., in [1]. For FinFETs or nanowire devices, because of the 3D nature of the structure, it is a formidable task to solve the current-carrying Schrödinger equation directly, even with methods like NEGF [2].

In this work, we present a hybrid approach to the quantum mechanical (QM) simulation of 3D FinFET/nanowire structures, which captures the carrier confinement in the crosssection of the fin/wire and the ballistic (including tunneling) transport along the channel direction. The computation cost for a sub-20 nm FinFET is quite affordable. Compared with the DG structure, FinFETs shows a better scalability and $I_{\mathrm{on}} / I_{\text {off }}$ ratio. The scaling limit is also revealed (it all depends on the channel length/cross-section dimension aspect ratio). Another benefit of using 3D QM simulation is that without proper multidimension quantum transport modeling, the doping profile extracted based on measured electrical data may be grossly distorted and thus deemed unphysical.

\section{Model Description}

The 3D numerical model with quantum mechanics, which we have developed, is based on: 2D Schrödinger equation in the cross section, 1D NEGF along the channel direction, and 3D Poisson's equation in all real-space dimensions. In this method, the most important technique is to separate a 3D QM function into two parts: in the orthogonal section and along the channel. A simple numerical implementation of this technique is based on tensor-product mesh with finite difference method. Then, there are two different equations to solve, NEGF for Schrödinger equation with open boundaries on 
two ends of the channel and eigenvalue problem in the cross section. Compared to a complete $3 \mathrm{D}$ numerical simulation, this method reduces computational complexity considerably.

For the cross sections of channel, the 2D Schrödinger equation is solved directly for eigenenergies and eigenwavefanctions of the Hamiltonian. Since in every slice, the wavefunction is confined in the device region, so the energy level, which can be called "mode energy", is discrete. The shape of the cross section can be arbitrary and the limit is that the cross section varies gradually along the channel. The density of states corresponding to three lowest modes in a rectangle slice are shown in Fig. 1. The confinement in the region causes disjoint peaks in the density of states for each mode.
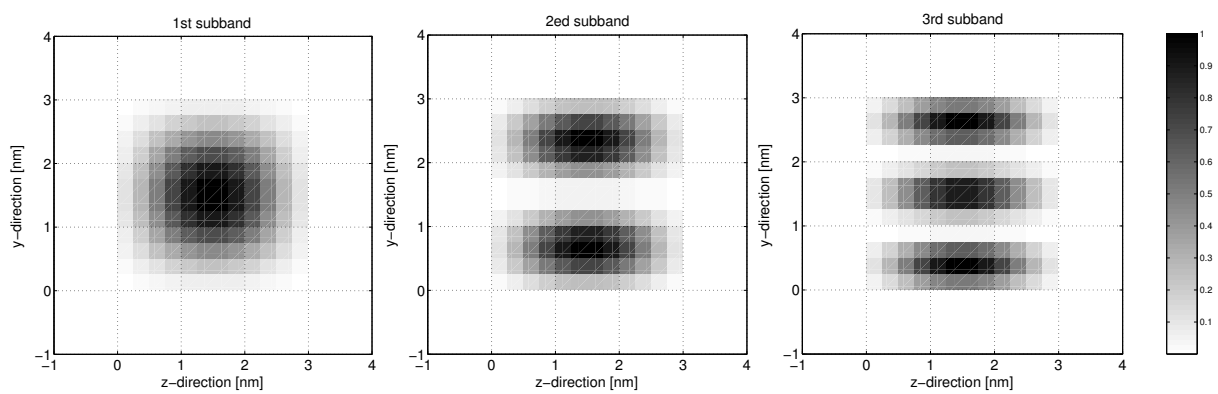

Figure 1: Density of states in cross section for three lowest subbands. The peak value of the density is normalized to unity. The dimension of the cross-section for the silicon slab is $3 \times 3 \mathrm{~nm}^{2}$ and $t_{o x}=1 \mathrm{~nm}$. Bias is set to zero.

Treating the mode energy as the effective 1D potential energy, NEGF along the channel is solved for different mode separately. In this approach, we must understand that the total energy of particle can be separated into longitudinal kinetic energy and transverse potential energy, while the mode energy is the sum of these two parts. A self energy matrix is used to represent the disturbance due to the open boundary and scattering in the device region in NEGF method. It only has diagonal elements. From the density matrix, which is obtained form 1D NEGF, the density of states along the channel is calculated [2].

Constructing 3D density of states from the longitudinal density of state and wavefunctions in cross-section is straightforward: need only to multiply the former with the square of the amplitude of wavefunction. The particle density at this energy is then the product of the 3D density of states and the distribution function. The 3D positiondependent particle density is the summation of that at all energy levels in every mode (subband). The channel current can be obtained from the density matrix from NEGF. We show the electron density in several cross sections of different size in Fig. 2. It is noticeable that the electrons peak at the center of the cross-section when the silicon slab is quite narrow. When the slab becomes wider, the peak evolves to a crater, which validates the prophesy about quantum confinement by physical conceptions.

With the electron density we have calculated the Poisson's equation should be iterately solved. For Poisson's equation, the boundary conditions are set to be as follows: (1) At the gate contacts, the gate vacuum potential is determined from the gate bias voltage and workfunction of the contact materials. (2) At the source/drain contacts and other 

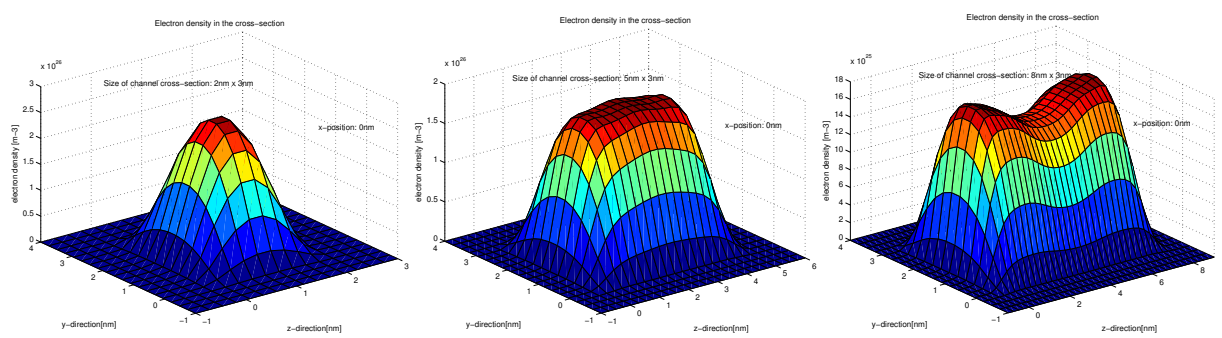

Figure 2: The profile of electron density for the cross sections at the begin of the channel $(x=0)$. The sizes of channel cross sections are $2 \times 3 \mathrm{~nm}^{2}$ (left), $5 \times 3 \mathrm{~nm}^{2}$ (middle), and $8 \times 3 \mathrm{~nm}^{2}$ (right). Bias is set to zero.

open boundarys, the natural Neumann boundary conditions are imposed, which permit potentials to float to whatever values necessary to ensure the charge neutrality at these boundaries [3]. By using Newton iterative method with "fixed" quasi-Fermi level this numerical implementation has a gratifying convergence behavior.

\section{Simulation Results and Discussion}

With this method, we have simulated several FinFET devices of different size. In Fig. 3, it is shown that the device with thinner slab has much higher threshold voltage. This is a reasonable result of tighter quantum confinement, which forces the inversion layer to swing away from the interface of channel and gate oxide. Since the tunnelling current increased greatly with the reduction of gate length, the threshold voltage decreases too, and the switch-off of channel becomes difficult. Here, we define the threshold voltage as the gate voltage when the channel current is $1 \mathrm{nA}$. Relative to this, thinner channel device has much better subthreshold slope characteristic and very low off-current.

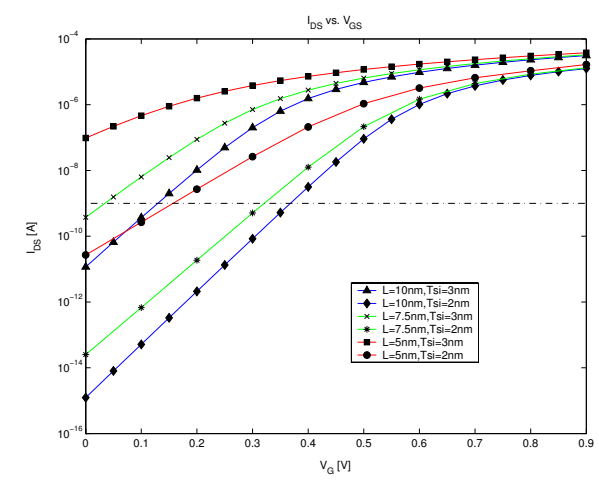

Figure 3: The $I_{D S}-V_{G S}$ curves for FinFET of $3 \times 3 \mathrm{~nm}^{2}$ and $2 \times 2 \mathrm{~nm}^{2}$. The oxide thickness is $1 \mathrm{~nm}$, and the gate length is $10 \mathrm{~nm}, 7.5 \mathrm{~nm}$ and $5 \mathrm{~nm}$. $V_{D S}=0.7 \mathrm{~V}$. Here we use the same gate work function $(4.05 \mathrm{eV})$ for all the devices.

Fig. 4 plots the the on-off current ratio of the devices as the channel length changes from 
$10 \mathrm{~nm}$ to $5 \mathrm{~nm}$. Here we define the on-current as the drain current when $V_{G}=0.9 \mathrm{~V}$ and $V_{D S}=0.7 \mathrm{~V}$, and the off-current as the drain current when $V_{G}=0$ and $V_{D S}=$ $0.7 \mathrm{~V}$. In this figure we also compare them with the simulation result of a double gate MOSFET [1]. Although the ratio of all devices is deteriorated with the decreasing gate length, the superiority of FinFET becomes more distinct. Benefited from its better gate control ability, FinFET has a much higher ratio than DG device, especially when the gate length is less than $10 \mathrm{~nm}$.

In Fig. 5, the DIBL characteristic of devices are plotted and the lower curve has a better performance. The two FinFET devices exhibit the same slope with the decreasing gate length, while DG has a higher slope. The curves shows that when channel dimensions equal to $10 \times 3 \times 3 \mathrm{~nm}^{3}$ and $7 \times 2 \times 2 \mathrm{~nm}^{3}$, the devices have similar performance. Therefore, to acquire acceptable device performance, retaining the channel length/cross-section dimension aspect ratio is essential.

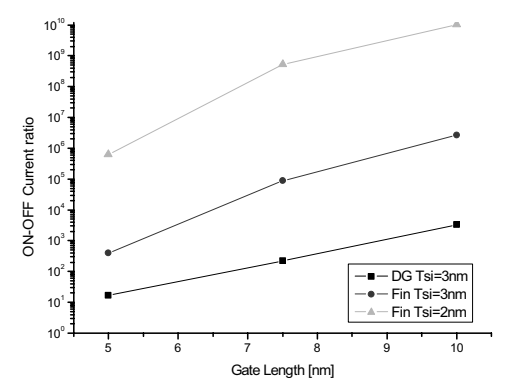

Figure 4: The device on-off current ratio vs. gate length curves for FinFETs and DG MOSFETs.

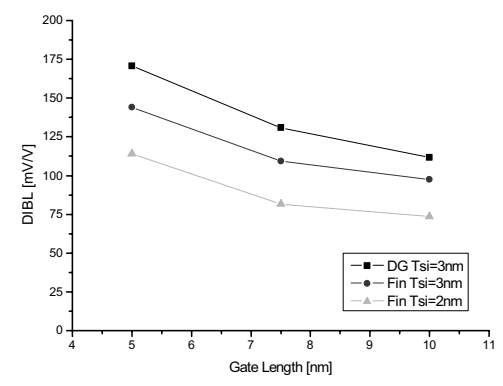

Figure 5: The device DIBL characteristic vs. gate length curves for FinFETs and DG MOSFETs.

\section{Conclusions}

With the quasi-3D numerical model we have developed, FinFET structures with ultrathin channel and gate oxide are simulated, the ballistic transport along the channel is accounted for by the application of NEGF. The results show that the nanoscale FinFET has an outstanding scalability beyond DG devices.

Acknowledgement: This work is supported by a grant from Ministry of Science and Technology (Plan 973, \#G200036502).

\section{References}

[1] Zhibin Ren, "Nanoscale MOSFETs: Physics, Simulation, and Design," pp. 55-64, Ph.D. thesis, Purdue University, West Lafayette, IN, USA, Dec. 2001.

[2] R. K. Lake and S. Datta, "The Non-equilibrium Greens function method applied to double barrier resonant tunneling diodes," Phys. Rev. B, vol. 45, pp. 6670-6685, 1992.

[3] G. W. Brown and B. W. Lindsay, "The numerical solution of Poisson's equation for twodimensional semiconductor devices," Solid-State Electronics, vol. 19, pp. 991-992, 1976. 